\title{
Safety and tolerability of therapeutic plasma exchange in autoimmune neurological diseases - a retrospective single-centre analysis
}

\author{
Agnieszka Gala-Błądzińska ${ }^{1,3}$, Katarzyna Mazur ${ }^{1}$, Adam Dębiec ${ }^{2}$, Krzysztof Gargasz $^{3}$, \\ Halina Bartosik-Psujek ${ }^{2,3}$
}

${ }^{1}$ Department of Internal Medicine, Nephrology and Endocrinology, St. Queen Jadwiga Clinical District Hospital No2

in Rzeszow, Rzeszow, Poland

${ }^{2}$ Neurology Clinic, St. Queen Jadwiga Clinical District Hospital No. 2 in Rzeszow, Rzeszow, Poland

${ }^{3}$ Medical College of Rzeszow University, Institute of Medical Sciences, Rzeszow, Poland

\begin{abstract}
Clinical rationale for study. The sudden onset of autoimmune neurological diseases often threatens life. In such clinical situations, fast, effective and safe treatment is needed. Therapeutic plasma exchange (TPE) is an option in the treatment of autoimmune disorders.
\end{abstract}

Aim of study. The aim was to assess the tolerability and safety of membrane-based therapeutic plasma exchange (mTPE) in patients with autoimmune neurological diseases.

Materials and methods. A total of 410 TPE treatments were performed in 91 adult patients. The main reasons for performing TPE were: Guillain-Barre syndrome (39.56\%), chronic inflammatory demyelinating polyradiculoneuropathy (20.88\%), and myasthenia gravis (17.58\%).

Results. A total of 183 (44.6\%) mTPE treatments were performed without complications. In 18 (19.8\%) patients, there were no complications observed in any of the MTPE procedures (a total of 83 procedures). Serious and life-threatening complications occurred during four (0.97\%) mTPEs. The most common abnormality in laboratory tests was hypocalcaemia. In patients with a fibrinogen concentration $\geq 2.63 \mathrm{~g} / \mathrm{L}$, measured before the second plasmapheresis, coagulation in the TPE filter was more frequently observed $(p=0.04)$. Conclusions and clinical implications. Our study proves that the use of plasmapheresis conducted by filtration in the treatment of autoimmune neurological diseases is safe and well tolerated.

Key words: autoimmune neurological diseases, therapeutic plasma exchange, efficacy, side effects

(Neurol Neurochir Pol 2020; 54 (4): 344-349)

\section{Introduction}

Therapeutic plasma exchange (TPE) is a clinical procedure for the replace of plasma, which aims to remove the immune system's extracellular components and the proinflammatory agents from the blood. These factors are of key importance in the pathogenesis of many diseases, including those affecting the nervous system. During TPE, the patient's plasma is separated and removed. Along with the patient's plasma, we remove specific, potentially pathogenic factors such as: antibodies $(\mathrm{Ag})$ including alloantibodies, monoclonal protein, circulating immune complexes, complement activation products, cytokines, atherogenic substances, and toxic compounds. TPE is thought to affect the immune system by: improving the function of the reticuloendothelial system; removing inflammatory mediators; changing antigen proportions and antibodies leading to the formation of better soluble immune complexes; and stimulating a lymphocyte

Address for correspondence: Agnieszka Gala-Błądzińska, Dialysis Centre, St. Queen Jadwiga Clinical District Hospital No. 2 in Rzeszów, Lwowska 60 St., 35-301 Rzeszów, Poland, e-mail: agala.edu@gmail.com 
Table 1. Indications for PTE in our study, and category and grade recommendations for TPE according to the ASFA [2]

\begin{tabular}{|c|c|c|c|c|}
\hline Neurological disorders & Indication & Category & Grade & $\begin{array}{l}\text { Number/percentage } \\
\text { of treated patients }\end{array}$ \\
\hline Acute disseminated encephalomyelitis & Steroid refractory & II & $2 \mathrm{C}$ & $7 / 7.69$ \\
\hline $\begin{array}{l}\text { Acute inflammatory demyelinating } \\
\text { polyradiculoneuropathy(Guillain-Barre syndrome) }\end{array}$ & Primary treatment & I & $1 \mathrm{~A}$ & $36 / 39.57$ \\
\hline $\begin{array}{l}\text { Chronic inflammatory demyelinating polyradiculoneuro- } \\
\text { pathy }\end{array}$ & $\begin{array}{l}\text { Severe relapse or steroid } \\
\text { refractory }\end{array}$ & I & $1 \mathrm{~B}$ & $19 / 20.89$ \\
\hline Multiple Sclerosis & Rapidly evolving MS & III & $2 \mathrm{~B}$ & $10 / 10.99$ \\
\hline Myasthenia gravis & $\begin{array}{l}\text { Moderate-to-severe } \\
\text { symptoms }\end{array}$ & I & $1 \mathrm{~B}$ & $16 / 17.58$ \\
\hline Neuromyelitis optica spectrum disorders & $\begin{array}{c}\text { Severe relapse or steroid } \\
\text { refractory }\end{array}$ & $\|$ & $1 \mathrm{~B}$ & $3 / 3.3$ \\
\hline
\end{tabular}

ASFA — American Society for Apheresis

population sensitised to cytotoxic treatment with which the plasma exchange is often associated. TPE also allows for supplementing the deficiencies of specific plasma factors [1-2]. In the current American Society for Apheresis (ASFA) guidelines, therapeutic plasma exchange is recommended for the treatment of 157 conditions. Disorders of the nervous system include 25 disease entities. TPE is most commonly used in Guillain-Barre syndrome (GBS), neuromyelitis optica spectrum disorders (NMOSD), myasthenia gravis (MG), Multiple Sclerosis (MS), and chronic inflammatory demyelinating polyradiculoneuropathy (CIDP) [2]. Also, in the latest update to the guidelines, two out of six new diseases listed are neurological or possibly involve the nervous system: amyotrophic lateral sclerosis, and POEMS syndrome (POEMS = polyneuropathy, organomegaly, endocrinopathy, monoclonal protein, skin changes) [2]. In the ASFA recommendations, indications for TPE are divided into four therapeutic categories, the first being where TPE treatment is the basic and preferred method of therapy, and the fourth category being where the use of TPE is ineffective and may even be harmful. In our dialysis centre, therapeutic plasma exchange is mainly performed in patients with autoimmune neurological diseases.

\section{Clinical rationale for the study}

The aim of this study was to assess the tolerability and safety of membrane-based therapeutic plasma exchange (mTPE) in patients with autoimmune neurological diseases.

\section{Materials and methods}

Our study involved patients treated with therapeutic plasma exchange for neurological indications between March 2013 and October 2017. In most of the disease entities, the TPE treatment was combined with pharmacotherapy in accordance with current recommendations. The data was assessed retrospectively based on the patients' medical records. The number and percentage of patients treated with TPE classified by category and the ASFA TPE grading recommendations are presented in Table 1 [2].

TPE was performed by filtration (mTPE) using the Gambro Prismaflex apparatus. Vascular access was obtained by introducing a two-lumen vascular catheter into the internal jugular, subclavian or femoral veins using the Seldinger technique. In all cases, cannulas sized $13.5 \mathrm{Fr} / 15 \mathrm{~cm}$ or $13.5 \mathrm{Fr} / 20 \mathrm{~cm}$ were inserted. For the TPE treatments, ready-made sets of capillary tubing and plasma filters from Gambro Prismaflex TPE 2000 were used, and prior to the procedure these were filled with saline and vented. Therapeutic plasma exchange was usually performed every two days. The duration of any single treatment did not exceed four hours. The number and mode of treatments depended on the clinical status of the patient and their underlying condition. On average, patients received both 1,000-1,500 mL of crystalloids (multi-electrolyte fluid, $0.9 \% \mathrm{NaCl}$ solution) and $1,000-1,500 \mathrm{~mL}$ of colloids. The total volume of plasma to be exchanged (EPV) was calculated based on the patient's haematocrit (Hct) level and body weight using the Kaplan formula:

$\mathrm{EPV}=[0.065 \times$ body weight $(\mathrm{kg})] \times[1-\mathrm{Hct}]$.

Where anticoagulation was required, unfractionated heparin was used, with an initial dose of $50 \mathrm{IU} / \mathrm{kg}$ body weight (2,000-3,000 IU on average) followed by a continuous intravenous infusion of unfractionated heparin at a dose of $1,000 \mathrm{IU} /$ /hour. In patients showing a severe clinical condition, with an increased risk of bleeding complications, or in the case of repeated problems with plating of the plasma filter, 2-3 activated-clotting time (ACT) measurements were taken at the bedside. ACT values were kept between 90-130 s. The blood flow rate was kept at $100-110 \mathrm{ml} / \mathrm{min}$, and transmembrane pressure (TMP) was within 20-60 mmHg. During mTPE, the patient's vital signs (blood pressure, heart rate using continuous ECG recording, oxygen saturation, frequency and character of breathing, body temperature, state of consciousness) and any possible side effects were closely monitored. In addition, the patient's skin layers were observed for the presence of urticaria or erythema. The technical parameters of the procedure were regularly monitored: TMP, arterial pressure, venous pressure, 
ultrafiltration, blood flow, and flow and temperature of substitution fluids.

In accordance with the protocols adopted by our dialysis centre, before the second mTPE, patients' blood counts, prothrombin time expressed as the international normalised ratio (INR), activated partial thromboplastin time (APTT), fibrinogen concentrations, potassium, magnesium, total protein, and serum albumin were each recorded and evaluated.

Complications observed during MTPE were divided using the World Apheresis Association (WAA) categories into mild (self-limiting without treatment), medium (requiring additional drugs during TPE), severe (being the reason for discontinuing the TPE treatment), and death [3].

\section{Statistical analysis}

Data was presented as the number of patients (the percentage of the group) for nominal variables, and as a median (lower-upper quartile). Distribution normality was assessed using the Shapiro-Wilk test. Where normality assumptions were not met, the differences between the two groups were tested using the Mann-Whitney U test. The Wilcoxon pairs order test was used to show the effect of the procedure on the patients' conditions. The dependence between the type of substitution fluid and the appearance of a clot in the dialysis set was demonstrated using the Chi-square independence test with the Yetsa correction. For the ROC curve analysis, cut-off values optimizing the Youden index were calculated. The predictability of clotting in the therapeutic plasma exchange kit was determined by the fibrinogen level. Results were statistically significant at $\mathrm{p}<0.05$. The Statistica 13.1 package (StatSoft) was used in the calculations.

\section{Results}

Over the course of 56 months, 410 mTPEs were performed in 91 patients aged between 18 and 88 years (mean 53.9). Men comprised 58 of the patients, women 33 .

In the group of patients who qualified for MTPE, the following comorbidities were most often observed: hypertension ( $\mathrm{n}=43 ; 47.25 \%)$, osteoarthritis $(\mathrm{n}=17 ; 18.68 \%)$, diabetes or impaired glucose tolerance $(\mathrm{n}=12 ; 13.19 \%)$, current or history of cancer ( $\mathrm{n}=11 ; 12.1 \%)$, hypothyroidism $(\mathrm{n}=11 ; 12.1 \%)$, history of Lyme disease $(n=10 ; 10.9 \%)$, and infections of the urinary or respiratory systems $(n=9 ; 9.89 \%)$. Other diseases, such as hypercholesterolemia, chronic kidney disease, peripheral osteoarthritis, vascular damage to the central nervous system, liver damage, obesity, epilepsy, chronic pancreatitis, and atrial fibrillation were observed in less than $10 \%$ of patients.

The number of procedures performed in one patient ranged from 1-7 (average 4.5). The patient who underwent one MTPE developed massive allergic symptoms after the albumin solution was transfused. The largest group, consisting of almost $1 / 3$ of the patients treated with MTPE, were those with Guillain-Barre syndrome. Table 1 shows the number and percentage of patients treated for neurological disorders.
A total of 183 (44.6\%) mTPE treatments were performed without complications. In $18(19.8 \%)$ patients there were no complications observed in any of the mTPE procedures (a total of 83 procedures). In the remaining patients, at least one $\mathrm{mTPE}$ procedure resulted in one or more complications.

We observed mild complications in $44(48.3 \%)$ patients during 80 (19.5\%) mTPE procedures, and moderate complications, requiring the administration of additional drugs, in $76(83.5 \%)$ patients during $119(29 \%)$ procedures. Table 2 presents the type, number and percentage of complications during TPE, with their severity measured according to WAA [3]. Of the 96 patients undergoing TPE, three (3.2\%) died. Deaths were a consequence of a severe course of the underlying disease (diffuse inflammation of the brain and spinal cord in two patients) or complications of the underlying disease (in one patient a myasthenic crisis with sepsis resulting from massive pneumonia). All deaths occurred long after the end of mTPE treatments, and were not related to the therapeutic plasma exchange.

After analysing the results of tests taken before the second TPE, we prepared Table 3, in which we identified the irregularities found in the test results along with the frequency of their occurrence in the studied population. In addition, it was observed that in patients with fibrinogen concentration $\geq 2.63 \mathrm{~g} / \mathrm{L}$, measured before the second plasmapheresis, coagulation in the TPE filter was more frequently observed $(p=0.04)$. The observed regularity is shown in Figure 1. Changes in other blood parameters did not significantly affect the complications associated with the TPE technique.

There was no correlation between age and complications related to mTPE in the study group $(\mathrm{p}<0.05)$. No correlation was observed between the conditions that are an indication for TPE and the complications observed during MTPE.

Technical problems with the catheter were observed significantly more frequently in patients with concomitant Lyme disease $(p=0.005)$, and paresthesia during mTPE was more common in patients with vascular CNS damage $(\mathrm{p}=0.05)$.

\section{Discussion}

Our work is one of few original papers to have summarised the use of therapeutic plasma exchange in neurological disorders [4-6]. The work presented here is the result of a five-year period of conducting therapeutic plasma exchange by the filtration method in our dialysis centre. In our study, most of the indications for TPE were in the first and second categories according to the ASFA recommendations [2].

TPE is an invasive method of treatment and carries a risk of complications, but in most cases these are mild; in our study, their frequency was estimated at $18.6 \%$. This percentage is much higher than in WAA data, in which mild complications occurred in $2.8 \%$ of TPE treatments from all indications; although in the WAA report only $17 \%$ of patients had neurological disorders [3]. In addition, some of the complications 
Table 2. Type, number and percentage of complications with severity according to WAA grading scale in course of TPE treatment

\begin{tabular}{|c|c|c|c|}
\hline $\begin{array}{c}\text { Grading of adverse } \\
\text { event }\end{array}$ & Symptoms/findings & $\begin{array}{c}\text { Patients with } \\
\text { complications (n/\%) }\end{array}$ & $\begin{array}{c}\text { TPE with } \\
\text { complications (n/\%) }\end{array}$ \\
\hline \multirow[t]{2}{*}{ Mild } & Hypotension not requiring replacement of pressure amines & $31 / 34.1$ & $64 / 15.6$ \\
\hline & Technical problems with the catheter & $13 / 14.28$ & $16 / 3.9$ \\
\hline \multirow[t]{16}{*}{ Moderate } & TMP increase or coagulation in the filter & $21 / 23.98$ & $21 / 5.12$ \\
\hline & Hypotension associated with plasma turning & $12 / 13.2$ & $35 / 8.5$ \\
\hline & Dyspnoea & $12 / 13.2$ & $18 / 4.4$ \\
\hline & Paresthesia & $7 / 7.7$ & $15 / 3.7$ \\
\hline & Chest pain & $4 / 4.4$ & $5 / 1.2$ \\
\hline & Headache & $4 / 4.4$ & $7 / 1.7$ \\
\hline & Back pain (spine) & $3 / 3.3$ & $4 / 1.0$ \\
\hline & Shivers, fever & $3 / 3.3$ & $4 / 1.0$ \\
\hline & Urticaria & $3 / 3.3$ & $3 / 0.7$ \\
\hline & Abdominal pain & $1 / 1.1$ & $1 / 0.2$ \\
\hline & Lower limb pain & $1 / 1.1$ & $1 / 0.2$ \\
\hline & Eye irritation, tears & $1 / 1.1$ & $1 / 0.2$ \\
\hline & Arrythmia - tachycardia & $1 / 1.1$ & $1 / 0.2$ \\
\hline & Arrythmia - bradycardia & $1 / 1.1$ & $1 / 0.2$ \\
\hline & Anxiety & $1 / 1.1$ & $1 / 0.2$ \\
\hline & Nausea, vomiting & $1 / 1.1$ & $1 / 0.2$ \\
\hline \multirow[t]{4}{*}{ Severe } & TRALI & $1 / 1.1$ & $1 / 0.2$ \\
\hline & Acute anaphylactic reaction & $1 / 1.1$ & $1 / 0.2$ \\
\hline & Sepsis staphylococcus & $1 / 1.1$ & $1 / 0.2$ \\
\hline & HIT II & $1 / 1.1$ & $1 / 0.2$ \\
\hline Death & - & 0 & 0 \\
\hline
\end{tabular}

HIT II - heparin-induced thrombocytopenia type II;TMP — transmembrane pressure; TRALI — transfusion-related acute lung injury; WAA — World Apheresis Association

Table 3. Irregularities in laboratory tests taken before second TPE

\begin{tabular}{|c|c|}
\hline Type of irregularity & $\begin{array}{c}\text { Number and percentage of patients }(n / \%) \text { with } \\
\text { laboratory irregularities }(n=78)\end{array}$ \\
\hline Anaemia $\left(\mathrm{Hgb}^{\dagger}<12 \mathrm{~g} / \mathrm{dL}\right.$ for women and $<13.0 \mathrm{~g} / \mathrm{dL}$ for men) & $28 / 29.17$ \\
\hline Leukopenia (leucocytes $<4,000 / \mu$ ) & $1 / 1.04$ \\
\hline Elevated leucocytosis (leukocyte count $>11,000 / \mu$ ) & $41 / 42.7$ \\
\hline Thrombocytopenia (platelet count $<100,000 / \mu$ ) & $14 / 14.58$ \\
\hline Hypokalaemia (serum potassium $<3.5 \mathrm{mml} / \mathrm{L}$ ) & $6 / 6.25$ \\
\hline Hyponatraemia (serum sodium $<135 \mathrm{mmol} / \mathrm{L}$ ) & $21 / 21.87$ \\
\hline Hypocalcaemia (decrease in total serum calcium < 2.25 mmol/L) & $49 / 51.04$ \\
\hline Hypomagnesaemia (magnesium concentration $<0.65 \mathrm{mmol} / \mathrm{L}$ ) & $21 / 21.87$ \\
\hline Lowered fibrinogen concentration $(<1.8 \mathrm{~g} / \mathrm{L})$ & $16 / 16.66$ \\
\hline $\mathrm{eGFR}^{\ddagger}<60 \mathrm{~mL} / \mathrm{min} / 1.73 \mathrm{~m}^{2}$ & $5 / 5.2$ \\
\hline Reduced total protein levels $<66 \mathrm{~g} / \mathrm{L}$ & $75 / 96.15$ \\
\hline Decreased albumin level $<35 \mathrm{~g} / \mathrm{L}$ & $12 / 12.5$ \\
\hline $\mathrm{INR}^{\S}<0.8$ or $>1.2$ & $2 / 2.08$ \\
\hline APTT $^{n}<24$ s or $>36 s$ & $10 / 10.42$ \\
\hline
\end{tabular}

${ }^{\dagger}$ Haemoglobin; " estimated Glomerular Filtration Rate; International Normalised Ratio; 'Activated Partial Thromboplastin Time 


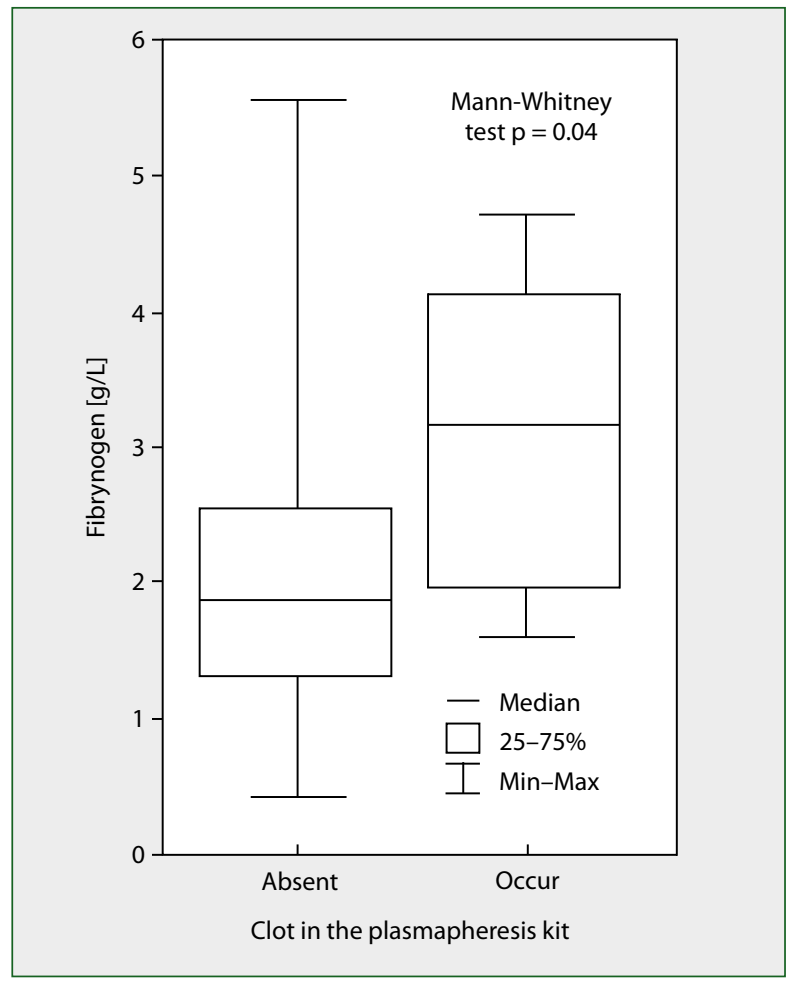

Figure 1. The effect of blood fibrinogen on the presence of clots in a TPE kit

that occur can be included in patients' subjective feelings, such as: pain in various places, paresthesia, or pruritus, and these may actually result from the course of the underlying disease and thus affect the higher rate of TPE complications that we observed.

Our study confirmed the findings of earlier reports that patients who undergo albumin replacement therapy are more likely to have pressure drops during TPE than those in whom FFP was used in the substitution [3]. In our patients, severe life-threatening complications accompanied only four treatments (0.97\%). In the WAA report, severe complications after apheresis occurred in less than $1 \%$ of cases, which confirms that it is a safe method [3]. The most common abnormalities in laboratory tests were hypocalcaemia caused, inter alia, by the binding of calcium ions by citrate, which is a component of fresh frozen transfusion plasma, or the removal of calcium from the vascular bed as a consequence of the plasma exchanging with liquids that either do not contain, or have a low concentration of, calcium ions. Hypocalcaemia lowers the cell excitability threshold and may be responsible for several tetany and paraesthesia symptoms. During mTPE conducted in our centre, symptoms that may be a consequence of hypocalcaemia were usually slightly intensified, but they could cause the patient discomfort or anxiety. Due to reports of frequent complications in the routine use of calcium carbonate in the prophylaxis of hypocalcaemia accompanying mTPE [3], in 2016 our centre ceased routine calcium substitution. Despite the lack of this, we did not find any increased symptoms that may be related to hypocalcaemia.

In our study, the next most frequently observed abnormality in the laboratory tests, observed in just over $40 \%$ of patients, was increased leucocytosis, which is probably related to the higher incidence of infection in this group of patients who were burdened with immune disorders resulting from the primary disease process and numerous co-morbidities. The immunosuppressive treatment used in some patients undergoing TPE treatment, including systemic glucocorticoids, is also important. An important role in the decline in immunity in patients treated with therapeutic plasma exchange is played by the depletion of immunoglobulins and the complement component C3-C4 [3, 7].

All the factors described above help explain the relatively higher frequency of septic complications occurring in this group of patients. We suggest that both laboratory tests and increased and reliable observations of the patient are required to look for signs of infection.

In our study, we observed that if the patient started a second mTPE with a fibrinogen concentration equal to or higher than $2.63 \mathrm{~g} / \mathrm{L}$, then he or she was more likely to clot in the filter during mTPE. In addition, it is important that the removal of a significant amount of antithrombin and other anticoagulants from the body can result in venous thromboembolism including pulmonary embolism [2]. Our work is probably the first to draw attention to the value of fibrinogen on the course of the MTPE procedure. In our work, unfractionated heparin was used as an anticoagulant during TPE treatment. However, we are aware of reports in which the authors describe the possibility of using TPE with filtration without using an anticoagulant [7-9]. For instance, Gashti et al. reported a 7.7\% incidence of clotting of the filter requiring cartridge/filter replacement, and they suggested that TMP should be closely monitored [8].

The pathogenesis of adverse reactions during TPE should consider the lower efficacy of pharmacotherapy in patients treated with therapeutic plasma exchange. Drugs that are extensively removed during therapeutic plasma exchange include those characterised by low distribution volume and strong protein binding such as: acetylsalicylic acid, cefazolin, ceftriaxone, chlorpropamide, diclofenac, heparin, ibuprofen, valproic acid, warfarin, and thyroxine [10]. Considering the above observations, it is usually recommended that all scheduled drugs should be administered immediately after the therapeutic plasma exchange procedure [11].

\section{Clinical implications}

TPE conducted by filtration is relatively safe in the treatment of neurological disorders, and the benefits of its use in autoimmune disease outweigh the risk of complications. Fibrinogen concentration $\geq 2.63 \mathrm{~g} / \mathrm{L}$, measured before the second TPE, significantly predisposes to the appearance of clotting 
in the TPE kit. Complications observed during TPE may be related to concomitant diseases that are not indications for TPE.

Acknowledgements: We thank Dr. Łukasz Budziak (St. Queen Jadwiga Clinical District Hospital No2 in Rzeszów) and Maciej Bładziński (Medical College of Rzeszow University) for help in data collection.

Ethical permission: Not necessary for the preparation of this article.

Funding: This article was prepared without any external source of funding.

Conflict of interest: The authors declare no conflict of interest or financial relationship.

\section{References}

1. Sokolov AA, Solovyev AG. Russian pioneers of therapeutic hemapheresis and extracorporeal hemocorrection: 100-year anniversary of the world's first successful plasmapheresis. Ther Apher Dial. 2014; 18(2): 117-121, doi: 10.1111/1744-9987.12067, indexed in Pubmed: 24571429.

2. Padmanabhan A, Connelly-Smith L, Aqui N, et al. Guidelines on the Use of Therapeutic Apheresis in Clinical Practice - Evidence-Based Approach from the Writing Committee of the American Society for Apheresis: The Eighth Special Issue. J Clin Apher. 2019; 34(3): 171-354, doi: 10.1002/jca.21705, indexed in Pubmed: 31180581.

3. Mörtzell Henriksson M, Newman E, Witt V, et al. Adverse events in apheresis: An update of the WAA registry data. Transfus Apher Sci. 2016; 54(1): 2-15, doi: 10.1016/j.transci.2016.01.003, indexed in Pubmed: 26776481.
4. Jani-Acsadi A, Lisak RP. Myasthenic crisis: guidelines for prevention and treatment. J Neurol Sci. 2007; 261(1-2): 127-133, doi: 10.1016/j.jns.2007.04.045, indexed in Pubmed: 17544450.

5. Guptill JT, Oakley D, Kuchibhatla M, et al. A retrospective study of complications of therapeutic plasma exchange in myasthenia. Muscle Nerve. 2013; 47(2): 170-176, doi: 10.1002/mus.23508, indexed in Pubmed: 23168720.

6. Weinshenker BG, O'Brien PC, Petterson TM, et al. A randomized trial of plasma exchange in acute central nervous system inflammatory demyelinating disease. Ann Neurol. 1999; 46(6): 878-886, doi: 10.1002/1531-8249(199912)46:6<878::aid-ana10>3.0.co;2-q, indexed in Pubmed: 10589540.

7. Wood L, Jacobs P. The effect of serial therapeutic plasmapheresis on platelet count, coagulation factors, plasma immunoglobulin, and complement levels. J Clin Apher. 1986; 3(2): 124-128, doi: 10.1002/ jca.2920030209, indexed in Pubmed: 3516979.

8. Gashti CN, Andreoli DC, Patel D. Membrane-based therapeutic plasma exchange (mTPE): Technical and clinical experience. J Clin Apher. 2018; 33(1): 38-45, doi: 10.1002/jca.21561, indexed in Pubmed: 28608569.

9. Córdoba JP, Larrarte C, Medina MC. Experience in therapeutic plasma exchange by membrane filtration at an academic center in Colombia: Registry of the first 500 sessions. J Clin Apher. 2015; 30(6): 347-352, doi: 10.1002/jca.21391, indexed in Pubmed: 25708454.

10. Ibrahim RB, Liu C, Cronin SM, et al. Drug removal by plasmapheresis: an evidence-based review. Pharmacotherapy. 2007; 27(11): 1529-1549, doi: 10.1592/phco.27.11.1529, indexed in Pubmed: 17963462.

11. Szczeklik W, Jankowski M, Wegrzyn W, et al. Acute respiratory failure in patients with Guillain-Barré syndrome and myasthenic crisis treated with plasmapheresis in the intensive care unit. Pol Arch Med Wewn. 2008; 118(4): 239-242, indexed in Pubmed: 18575425. 